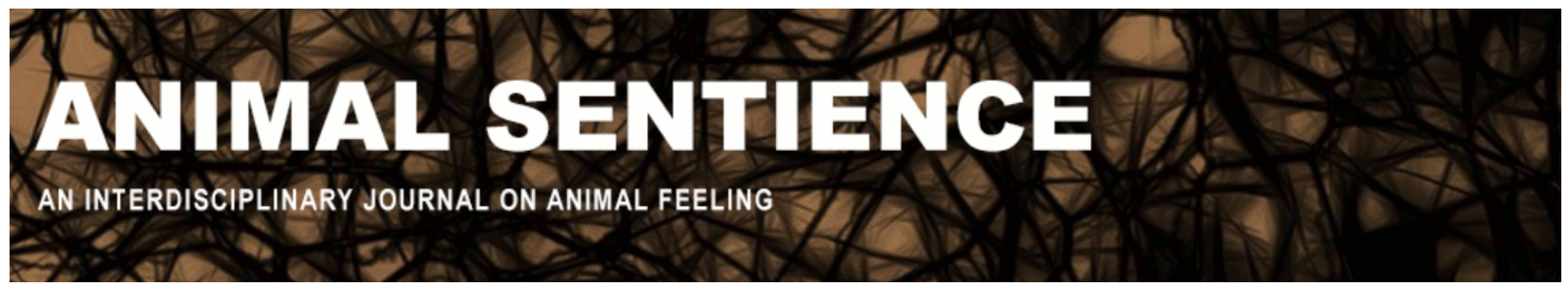

Kotler, Burt P. and Brown, Joel S. (2017) Fear and loathing on the landscape: What can foraging theory tell us about vigilance and fear?. Animal Sentience 15(5)

DOI: $10.51291 / 2377-7478.1280$

Date of submission: 2017-12-21

Date of acceptance: 2017-12-27

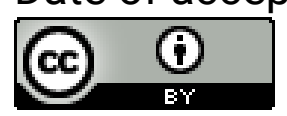

This article has appeared in the journal Animal

Sentience, a peer-reviewed journal on animal

cognition and feeling. It has been made open access,

free for all, by WellBeing International and deposited

in the WBI Studies Repository. For more information,

please contact

wbisr-info@wellbeingintl.org.

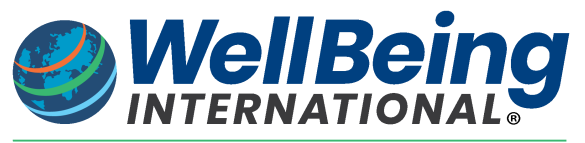

SOLUTIONS FOR PEOPLE, ANIMALS AND ENVIRONMENT 


\title{
Fear and loathing on the landscape: What can foraging theory tell us about vigilance and fear?
} Commentary on Beauchamp on Fear \& Vigilance

\author{
Burt P. Kotler \\ Ben-Gurion University of the Negev, Israel \\ Joel S. Brown \\ University of Illinois at Chicago \& Moffitt Cancer Center, Florida
}

\begin{abstract}
We discuss fear and vigilance from the perspective of foraging theory. Rather than focusing on proximate indicators of fear, we suggest that fear is an adaptation for assigning a cost to activities that incur a risk of injury or death. We use theory to provide definitions for fear and vigilance and then use that theory to compare them. We agree that there are limits to the reliability of vigilance as an indicator of fear, but we arrive at this conclusion differently.
\end{abstract}

Keywords: vigilance, food-safety tradeoffs, predation risk, fear, landscape of fear, foraging theory

Burt P. Kotler, Professor of desert ecology at BenGurion University of the Negev, studies behaviors and other traits of organisms as adaptations in the tradeoffs between food and safety. He applies this to foraging behavior, risk management, mechanisms of species coexistence and predator-prey foraging games. in.bgu.ac.il/en/bidr/SIDEER/MDDE/Pages/staff/burtkotler.aspx

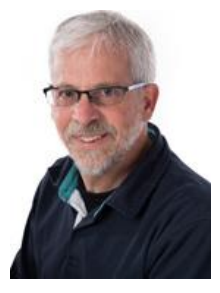

Joel S. Brown, Distinguished Professor Emeritus of Biological Sciences at the University of Illinois at Chicago, is an "optimist" who uses optimization and evolutionary game theory to understand ecological phenomena from cancer dynamics to intercontinental community convergence.

bios.uic.edu/bios/people/faculty/joel-brown

In his target article, Guy Beauchamp (2017) concludes that "vigilance may not always be a reliable indicator of the state of fear." We concur, but we arrive at this by a somewhat different route. The target article focuses on proximal indicators of fear such as stress hormones, heart rate, and other physiological and neurological responses. We suggest that fear is an adaptation for assigning a cost to activities that incur a risk of injury or death. Vigilance, stress hormones, physiological state, giving-up densities (the amount of food left behind in a resource patch following patch exploitation), and time allocation then become tools by which animals manage and respond to risk. An organism's use of these tools may covary positively, negatively, or not at 
all. It may even be optimal under some circumstances for an animal to exhibit more vigilance under safer rather than riskier conditions.

Beauchamp (2017) largely skips the question of theory and the optimal level of vigilance for foraging animals (starting with Pulliam, 1973), but he does consider them in Beauchamp (2015). Theory tells us how risk of predation can affect foraging decisions, including patch use and vigilance (e.g., Lima, 1996; Brown, 1988, 1999). When harvesting resources from depletable patches, an optimal forager should feed in a patch as long as its harvest rate in the patch exceeds the sum of its various foraging costs (Brown, 1988, 1992). It should harvest the patch as long as it profits from doing so. The gains of patch exploitation come from harvesting food. The costs arise from energetic costs of foraging, from the missed opportunity of not engaging in other valuable activities, and from exposure to risk of predation.

The risk of predation gives rise to a foraging cost, and animals respond to it by foraging less, avoiding risky habitats and microhabitats, directing their efforts to safer places, and altering their vigilance (e.g., Lima \& Dill, 1990; Brown \& Kotler, 2004). By doing so, foragers accept lower harvest rates and harvest less food. In return they lower their chances of being captured by a predator. This tradeoff of food and safety has consequences for population growth rates, species interactions, and community structure. The predation cost of foraging, $P$, can take the form (Brown, 1992):

$$
P=\frac{\mu \cdot F}{\frac{\partial F}{\partial e}} \quad \text { Eq. } 1
$$

where $P$ is the predation cost of foraging, $\mu$ is the risk of predation, $F$ is survivor's fitness (the fitness that a forager expects should it live to its next reproduction), and $\partial F / \partial e$ is the marginal value of energy (or resources) (Houston \& McNamara, 1999; Clark, 1994; Brown, 1992).

The "job" of fear is to assign a predation cost to an activity. The ecological manifestation of fear includes how much time an organism allocates to different times and places, and how much vigilance to employ while there. From this perspective, fear should be determined by the risk of predation, the energetic state of the organism, its prospects for the future, and the value of the current activity. Here we apply an ultimate causation definition for fear as an adaptation. It ignores the question of how a given species assesses and processes the key bits that go into $P$.

Our definition makes it easy to define the landscape of fear (Laundre at al. 2010). It is simply the predation costs of foraging mapped out in time and space across the physical landscape (van der Merwe \& Brown 2008). It can be mapped by measuring the quitting harvest rate of foragers and how it changes across the landscape. Such landscapes have been mapped for a variety of organisms including Cape ground squirrels (van der Merwe \& Brown 2008), ibex (Iribarren \& Kotler 2012a), klipspringers (Druce et al. 2006), gerbils (Bleicher et al. in review), and free-ranging goats (Shrader et al. 2008). Such maps reveal the features of the landscape that are most beneficial to organisms and those that are most detrimental. The landscape of fear has great utility in conservation and management.

Our definition avoids the pitfalls of what organisms are feeling emotionally, what brain processes are ongoing, whether their nervous systems are complex enough to feel fear, whether their physiological responses are consistent with their behavior, etc. We focus on fear as the product of natural selection. We feel that our definition has greater ecological relevance. It also 
opens up a much wider spectrum of organisms that we can consider to be fearful, from singlecell organisms to multi-cellular organisms that lack central nervous systems and even to plants. All that is necessary is that they can assess and respond behaviorally (including how they invest carbon) to risk and the other components required to estimate $P$.

Theory also informs us about the optimal use of vigilance (e.g., Lima, 1987; Brown, 1999). For cases when vigilance can reduce mortality risk, optimal vigilance for forager $i$ can be given by (extensions of this relationship exist for group foraging, Ale \& Brown, 2007; McNamara \& Houston, 1992):

$$
u_{i}^{*}=\sqrt{\frac{m_{i} F}{f_{i} b_{i}\left(\frac{\partial F}{\partial e}\right)}}-\frac{k_{i}}{b_{i}} \quad \text { Eq. } 2
$$

where $u_{i}{ }^{*}$ is the optimal vigilance level $\left(u_{i}^{*}=0\right.$, if (2) returns a negative value), $m_{i}$ is encounter rate with predators, $1 / k_{i}$ is the lethality of the predator, $b_{i}$ is the effectiveness of vigilance in reducing mortality, and $f_{i}$ is resource harvest rate for a non-vigilant forager. Vigilance rates should increase with higher encounter rates with predators, better future prospects, higher current energetic state (low marginal value of energy - hungry foragers should be less vigilant than well-fed ones), and greater lethality of the predator. Interestingly, the relationship with effectiveness of vigilance is inverted U-shaped. It reaches its highest values at intermediate levels of effectiveness. When vigilance is highly effective, a forager needs little; when it is ineffective, a forager wants to use little (Brown, 1999). Manipulations of sightlines in gerbils and in Nubian ibex confirm these predictions (Embar et al., 2011; Iribarren \& Kotler, 2012b).

Equations 1 and 2 quantify the relationship between fear and vigilance. They share three terms appearing in the same place in both equations: survivor's fitness, marginal value of energy, and predator encounter rate (predator encounter rate is a component of $\mu$ ). $F$ and $\partial F / \partial e$ are properties of the particular individual. They do not change as the forager moves from microhabitat to microhabitat and from habitat to habitat across the landscape. Foragers "carry" these values with them. The state and the marginal value of energy may vary with population, from week to week, perhaps from day to day, and from individual to individual. Vigilance will reflect these differences in fear.

Notice how these differences in vigilance do not yet indicate differences in encounter rates with predators. The encounter rate with predators can change from hour to hour and from habitat to habitat across the landscape. These changes too will manifest in changes in an individual's vigilance level as it moves through its landscape. So, do vigilance levels provide a good surrogate for $P$ and/or predation risk in general? Yes and No. When the driver of vigilance is encounter rate with predators, and predator lethality in the absence of vigilance, then: Yes. But when the driver is changes in the value of vigilance: then No. If vigilance is of little value to a forager in one habitat compared to another, there will be little vigilance by the forager in that habitat, whereas the other habitat will see higher vigilance levels; the latter is made safer by vigilance, but the former remains risky despite vigilance. It is precisely the value of vigilance that makes one riskier and the other safer. In this case, vigilance will covary negatively with mortality risk and perhaps with things like stress hormones, heart rate, and other physiological responses to risk. Can this happen? We have unpublished data suggesting that this is the case for grey squirrels and fox squirrels at sites around Chicago. The squirrels are less vigilant when foraging 
away from the trees (risky habitat) than near trees (safe habitat). Why? The closer a squirrel is to a tree, the more readily it can escape an encounter with a predator such as a hawk or coyote. Hence, the value of vigilance is greater near than far from a tree.

Another key aspect is that vigilance is just one tool - perhaps complementary, perhaps antagonistic, or perhaps substitutable - among other responses. Time allocation is another tool for managing predation risk. Foragers use both to determine their optimal patch use and givingup densities while exploiting depletable resource patches (Brown, 1999). Foragers can use a multitude of combinations of time allocation and vigilance strategies to arrive at a given givingup density. Under conditions where vigilance is ineffective, foragers may manage risk mostly with time allocation. Empirically, this is seen when they are distracted by the burden of fleas (Raveh, et al., 2011) or by vegetation that blocks sight lines (Embar, et al., 2011). In these cases, a mapping of the landscape of fear would look very different from a mapping of vigilance. The decoupling of fear and vigilance limit the ability to infer one from the other. In this manner, there are limits to vigilance reflecting fear. For a variety of applications, we see giving-up densities as a more useful tool for gaining insights into $P$ and actual predation risk.

The above perspectives emerge because we are ecologists, and keenly interested in the distribution and abundances of organisms. We focus on how behavior influences population dynamics and species interactions such as competition and predation. We are most interested in mechanisms of species coexistence, so we focus on foraging behaviors that mediate interactions between foragers and their resources (or prey) and with their predators. Predators can affect their prey numerically by killing them (direct effect), and they can affect them by scaring them and causing them to change behavior (i.e., indirect effect). Systems where the former predominate are $\mathrm{N}$-driven, and those where the later predominate are $\mu$-driven (often used by ecologists to represent the mortality risk of predation) (Brown \& Kotler, 2007). Effects arising from the risk of predation are sometimes called trait-mediated indirect effects; they were formerly referred to as higher-order species interactions and non-linear effects. Our focus on the predation costs of foraging allows us to best connect the foraging behavior of organisms to these processes and mechanisms. We fully appreciate approaches to vigilance that explore the neurological, hormonal, and physiological connections as cognitive animals attempt to assign $P$ and respond accordingly. Yet, as foragers assess and respond to risk, there will often be limits to how one particular tool of risk management reveals fear.

\section{References}

Ale, S. B., \& Brown, J. S. (2007). The contingencies of group size and vigilance. Evolutionary Ecology Research, 9, 1263-1276.

Beauchamp, G. (2015). Animal vigilance: monitoring predators and competitors. Academic Press, Amsterdam. 254 pp.

Beauchamp, G. (2017). What can vigilance tell us about fear? Animal Sentience 15(1).

Bleicher, S. S., Kotler, B. P., \& Brown, J. S. Comparing the landscapes of fear of four convergent rodent species under risk from owls and vipers. In review.

Brown, J. S. (1988). Patch use as an indicator of habitat preference, predation risk, and competition. Behavioral Ecology and Sociobiology, 22, 37-47. 
Brown, J. S. (1992). Patch use under predation risk: I. Models and predictions. Annales Zoologici Fennici, 29, 301-309.

Brown, J. S. (1999). Vigilance, patch use and habitat selection: foraging under predation risk. Evolutionary Ecology Research, 1, 49-71.

Brown, J. S., \& B. P., Kotler. (2004). Hazardous duty pay: studying the foraging cost of predation. Ecology Letters, 7, 999-1014.

Brown, J. S., \& B. P., Kotler. (2007). The ecology of fear. In: Foraging. Stephens, D., Ydenberg, R., \& Brown, J.S., eds. University of Chicago Press, Chicago.

Clark, C. W. (1994). Antipredator behavior and the asset-protection principle. Behavioral Ecology, 5, 159-170.

Druce, D. J., Brown, J. S., Castley, J. G., Kerley, G. I. H., Kotler, B. P., Mackey, R.L., \& Slotow, R. (2009). Spatial and temporal scaling in habitat utilization by klipspringers (Oreotragus oreotragus) determined using giving-up densities. Austral Ecology, 34, 577-587.

Embar, K., Kotler, B. P., \& Mukherjee, S. (2011). Risk management in optimal foragers: the effect of sightlines and predator type on patch use, time allocation, and vigilance in gerbils. Oikos, 120, 1657-1666.

Houston, A. I., \& McNamara, J. M. (1999). Models of adaptive behaviour: an approach based on state. Cambridge University Press.

Iribarren, C., \& Kotler, B. P. (2012a). Patch use and vigilance behaviour by Nubian ibex: the role of the effectiveness of vigilance. Evolutinary Ecology Research, 14, 223-234.

Iribarren, C., \& Kotler, B. P. (2012b). Foraging patterns of habitat use reveal landscape of fear of Nubian ibex Capra nubiana. Wildlife Biology, 18, 194-201.

Laundré, J. W., Hernández, L., \& Altendorf, K. B. (2001). Wolves, elk, and bison: reestablishing the "landscape of fear" in Yellowstone National Park, U.S.A. Canadian Journal of Zoology, 79, 1401-1409.

Laundré, J. W., Hernández, L., \& Ripple, W. J. (2010). The landscape of fear: ecological implications of being afraid. Open Ecology Journal, 3, 1-7.

Lima, S. L. (1987). Vigilance while feeding and its relation to the risk of predation. Journal of Theoretical Biology, 124, 303-316.

Lima, S. L. (1996). The influence of models on the interpretation of vigilance. In: Readings in animal cognition. MIT Press, Cambridge, MA. pp. 201-216.

Lima, S. L., \& Dill, L. M. (1990). Behavioral decisions made under the risk of predation - a review and Prospectus. Canadian Journal of Zoology-Revue Canadienne De Zoologie, 68, 619-640.

McNamara, J. M., \& Houston, A. I. (1992). Evolutionarily stable levels of vigilance as a function of group size. Animal Behaviour, 43, 641-658.

Pulliam, H. R. (1973). On the advantages of flocking. Journal of Theoretical Biology, 38, 419-422.

Raveh, A., Kotler, B. P., Abramsky, Z., \& Krasnov, B. R. (2011). Driven to distraction: detecting the hidden costs of flea parasitism through foraging behavior in gerbils. Ecology Letters, 14, 4751.

Shrader, A. M., Brown, J. S., Kerley, G. I. H., \& Kotler, B. P. (2008). Do free-ranging domestic goats show 'landscapes of fear? Patch use in response to habitat features and predator cues. Journal of Arid Environments, 72, 1811-1819.

van der Merwe, M., \& Brown, J. S. (2008). Mapping the landscape of fear of the Cape ground squirrel (Xerus inauris). Journal of Mammalogy, 89, 1162-1169. 